Welikala, R.A., Fraz, M.M., Habib, M.M., Daniel-Tong, S., Yates, M., Foster, P.J., Whincup, P.H., Rudnicka, A.R., Owen, C.G., Strachan, D.P. and Barman, S.A (2017) Automated quantification of retinal vessel morphometry in the UK Biobank Cohort. In Proceedings of the Seventh International Conference on Image Processing Theory, Tools and Applications IPTA 2017. Piscataway, U.S. : Institute of Electrical and Electronics Engineers, Inc. ISSN (online) 2154-512X ISBN 9781538618417

http://dx.doi.org/10.1109/IPTA.2017.8310108 


\title{
Automated Quantification of Retinal Vessel Morphometry in the UK Biobank Cohort
}

\author{
R.A. Welikala ${ }^{1}$, M.M. Fraz ${ }^{2}$, M.M. Habib ${ }^{1}$, S. Daniel-Tong ${ }^{3}$, M. Yates ${ }^{4}$, P.J. Foster ${ }^{5}$, P.H. Whincup ${ }^{6}$, A.R. \\ Rudnicka $^{6}$, C.G. Owen ${ }^{6}$, D.P. Strachan ${ }^{6}$, S.A. Barman ${ }^{1}$, on behalf of the UK Biobank Eye and Vision Consortium ${ }^{7}$ \\ ${ }^{1}$ School of Computer Science and Mathematics, Kingston University, Surrey, United Kingdom. \\ e-mail: r.welikala@kingston.ac.uk, s.barman@kingston.ac.uk \\ ${ }^{2}$ School of Electrical Engineering and Computer Science, National University of Sciences and Technology, Islamabad, Pakistan. \\ ${ }^{3}$ Department of Ophthalmology, North Middlesex University Hospital, London, United Kingdom. \\ ${ }^{4}$ Norwich Medical School, University of East Anglia, Norwich, United Kingdom. \\ ${ }^{5}$ NIHR Biomedical Research Centre, Moorfields Eye Hospital, London and UCL Institute of Ophthalmology, London, United Kingdom. \\ ${ }^{6}$ Population Health Research Institute, St. George's, University of London, London, United Kingdom. \\ ${ }^{7}$ Members of the UK Biobank Eye and Vision Consortium are listed before References.
}

\begin{abstract}
The morphometric characteristics of the retinal vascular network have been associated with risk markers of many systemic and vascular diseases. However, analysis of data from large population based studies is needed to help resolve uncertainties in these associations. QUARTZ (QUantitative Analysis of Retinal vessel Topology and siZe) is a fully automated retinal image analysis system that has been designed to process large numbers of retinal images and obtains quantitative measures of vessel morphology to be used in epidemiological studies. QUARTZ has been used to process retinal images from UK Biobank which is a large population-based cohort study. In this paper, we address issues of robustness with respect to processing large datasets and validate QUARTZ using a subset of 4,692 UK Biobank retinal images. Ground truth data produced by human observers for validation have been made available online. Following validation, 135,867 retinal images $(68,549$ participants) from the UK Biobank study were processed by QUARTZ. $71.53 \%$ of these images were classified as being of adequate quality, which equated to $80.90 \%$ participants with at least one image of adequate quality. The vessel morphometric data are currently being used in epidemiological studies. The intention of the UK Biobank Eye and Vision Consortium is to include these derived measures in the UK Biobank data archive.
\end{abstract}

Keywords-Retinal image analysis, UK Biobank, Large retinal datasets, Disease biomarkers.

\section{INTRODUCTION}

Cardiovascular disease (CVD) alone accounts for nearly 200,000 deaths in the UK per year [1]. CVD is also responsible for a substantial burden of morbidity and disability, accentuated by an ageing population and rising survival rates following myocardial infarction [2,3]. Therefore, early detection and prevention of CVD outcome is key. Diabetes and lesser degrees of hyperglycaemia are important determinants of CVD risk [4]. These precursors, along with other patient phenotypes, are used to estimate future risk of cardiovascular disease, providing indications for interventions to alter disease trajectory [5,6]. In addition to this, recent evidence suggests that biomarkers of the presence of CVD may improve risk prediction [7].

Examination of retinal images offers an opportunity to directly and non-invasively observe the blood circulatory system. The morphological characteristics of retinal vessels have been prospectively associated with cardiovascular and systemic disease [811]. Therefore, the accurate assessment of retinal vessel morphology may be an important biomarker of early vascular disease [12], which could enhance CVD risk prediction. However, some inconsistencies in the presence or absence of these associations remain [13,14]. This is perhaps due to uncertainty caused by sample size.
UK Biobank [15] contains probably the world's largest retinal image repository in a middle-aged population-based cohort study. Analysis of data from such a large study will help to resolve uncertainties when establishing the presence (or absence) of associations between the morphological characteristics of the retinal vascular network and future risk of cardiovascular and systemic disease. Computer automated measurements of vessels is required because images can be rapidly processed to obtain objective measures, unlike the manual equivalent which is subjective, open to error, labour intensive and therefore time consuming.

QUARTZ (QUantitative Analysis of Retinal vessel Topology and siZe) is a retinal image analysis system developed by our research group, which is an automated processing system for large numbers of retinal images and obtains quantitative measures of vessel morphology to be used in epidemiological studies [16]. Other notable systems exist, which include VAMPIRE [17-19], SIRIUS [20], ARIA [21], CAIAR [22,23] and SIVA [24]. However, to the best of our knowledge these systems have not been applied in a fully automated manner to the UK Biobank dataset. QUARTZ is fully automated in all aspects and information is extracted from the whole retina (not just concentric areas centred on the optic disc).

The fully automated framework of QUARTZ removes the need for manual intervention, which makes it feasible to run on the entire UK Biobank dataset. From our experience, most large retinal datasets used in epidemiological studies contain large amounts of poor quality images caused by a variety of issues. A particular problem is poor/uneven illumination of the retina due to avoiding the use of pharmacological mydriasis when capturing the images. Other issues including lens artefacts, eye lashes, media opacity, focal plane error and head/eye movement. UK Biobank camera operators received basic training/instructions and were not expert retinal photographers. Hence an automated system needs to be highly robust when processing a dataset with images of variable quality.

Algorithm adaptations to address issues of robustness in respect to large datasets are documented in this paper, along with an evaluation using a subset of 4,692 UK Biobank retinal images. Following this substantial validation, the entire retinal dataset $(135,867$ retinal images) from the UK Biobank study at baseline examination was automatically analysed using QUARTZ to produce vessel morphometric data. This marks an important milestone in the field of retinal image analysis, as to the best of our knowledge, we are the first group to achieve this task. The vessel morphometric data derived from QUARTZ is currently being used in epidemiological studies [25], with further findings to be published soon. 
The intention of the UK Biobank Eye and Vision Consortium is to include these data in the UK Biobank data archive. Therefore, the thorough validation of all aspects of QUARTZ on the UK Biobank retinal images is crucial in order to support all future studies that use these data.

\section{UK BIOBANK}

UK Biobank is a major national health resource with the aim of improving the prevention, diagnosis and treatment of a wide range of serious and life-threatening illnesses - including cancer, heart diseases, stroke, diabetes, arthritis, osteoporosis, eye disorders, depression and forms of dementia. UK Biobank recruited more than 500,000 people aged between 40-69 years in 2006-2010 from across the country. Participants underwent a physical examination (including retinal photography), provided blood, urine and saliva samples for future analysis, provided detailed information about themselves and agreed to have their health followed. The UK Biobank study was approved by the Northwest Region NHS research ethics committee.

A subset of 68,549 participants had retinal images captured, providing 135,867 macular centred retinal images (captured from both eyes for most of the participants). Colour images were captured with a non-mydriatic fundus camera (Topcon 3DOCT-1000 Mk 2) with a $45^{\circ}$ field-of-view and saved in PNG format with a resolution of $2048 \mathrm{x}$ 1536 pixels.

\section{QUARTZ OVERVIEW}

The QUARTZ software system was developed to provide epidemiologists with a fully automated framework for extracting quantitative measures of retinal vessel morphology from images obtained from large population based studies. Following the processing performed by QUARTZ, all measures are exported and saved as either CSV (comma separated values) or Excel files. The vessel analysis visualization screen of QUARTZ is illustrated in Fig.1.

The software was developed in Matlab using object oriented programming, inspired by [21]. This allows the software to be structured into modules which include vessel segmentation, vessel analysis, arteriole/venule $(\mathrm{a} / \mathrm{v})$ classification, optic disc localization and image quality assessment. Brief algorithm details of these modules are presented in this section; see $[16,26,27]$ for full details. This is accompanied by evaluation of the modules using a subset of 4,692 retinal images, attained from 2,346 random UK Biobank participants. 3,351 images were deemed to be of adequate image quality (explained in section E). All aspects of this subset were assumed to be representative of the whole of the UK Biobank dataset.

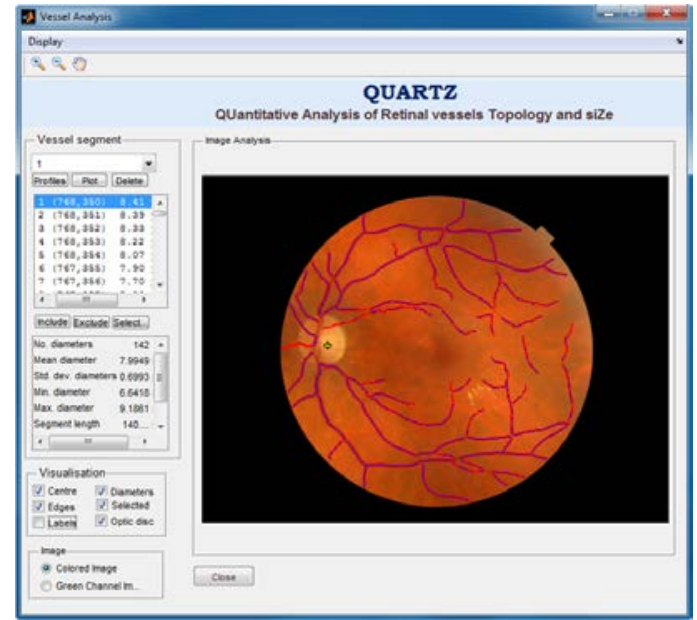

Fig.1. QUARTZ vessel analysis visualization screen.

\section{A. Vessel segmentation}

Retinal vessel segments can be approximated as being piecewise linear. In QUARTZ, we have implemented an unsupervised vessel segmentation approach based on a multi-scale line detector [28], linearly combining the line responses at varying scales to produce a final line strength image. This was followed with the application of a hysteresis thresholding based morphological reconstruction to produce the segmented vasculature (binary vessel map). Fraz [16] provides a more detailed description of this methodology. For epidemiological studies, the need to keep the segmentation of nonvessel objects to a minimum is more important than the segmentation of the complete vasculature. Therefore, additional steps to those documented in [16], of pre-processing (removal of bright intensities) and post-processing (masking out fovea and removal of small segmented objects) were performed to reduce false positives in the segmentation, documented in [26].

The automatic segmentations were compared with the manual segmentations by two human observers on a set of 20 retinal images. These 20 images were randomly selected from the 3,351 images of adequate quality. The segmentations of the first human observer were taken as the ground truth for the dataset. The comparison of the segmentations of the second human observer with the ground truth is regarded as the target performance level. The manual segmentations by both human observers have been made publicly available online (https://blogs.kingston.ac.uk/retinal/uk-biobank).

The results of the segmentation algorithm are shown in Fig.2 and are summarised in Table 1, achieving a similar accuracy to the $96.11 \%$ achieved by the 2nd human observer. The accuracy of $95.64 \%$, sensitivity of $73.66 \%$ and specificity $98.14 \%$ of the algorithm (without pre/post processing) are all comparable with state-of-the-art methods [29-31] which were applied on the datasets of DRIVE [32] and STARE [33]. This demonstrates the robustness of the algorithm, achieving this high level of performance despite the considerable variability in image quality of the UK Biobank dataset. The introduction of pre/post processing has the desired effect of increasing specificity by $0.74 \%$ (at the cost of sensitivity). This meets the requirements for epidemiological studies, in which it is vital to keep the segmentation of non-vessel objects to a minimum.
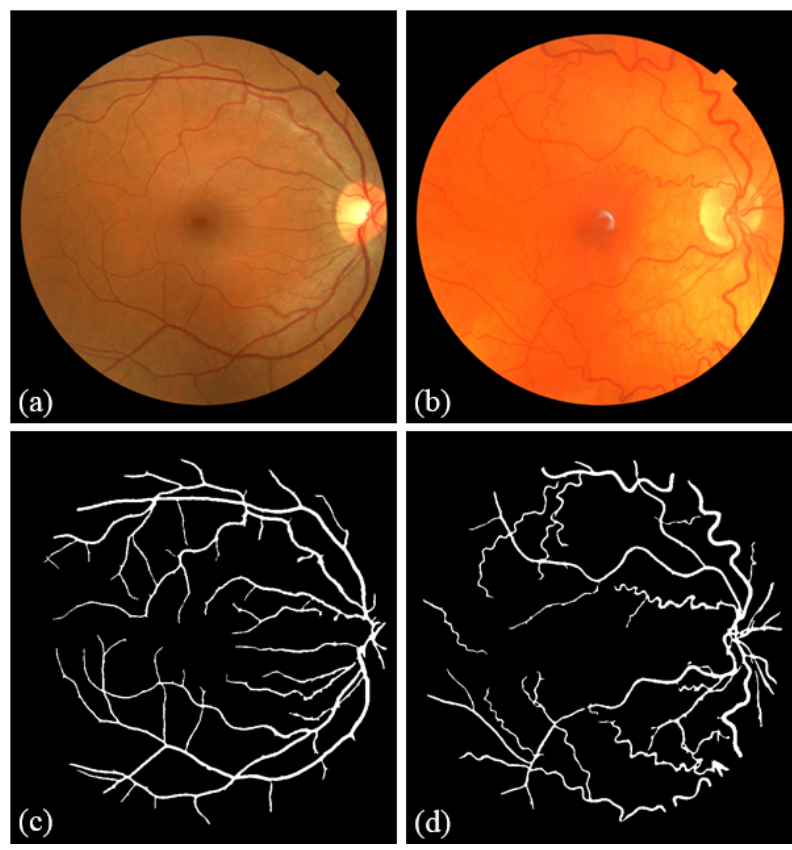

Fig.2. (a)-(b) Colour retinal images. (c)-(d) Segmentation of (a)-(b). (c) UK Biobank. 
Table 1. Performance of vessel segmentation (per pixel basis).

\begin{tabular}{|c|c|c|c|}
\hline Method & Accuracy & Sensitivity & Specificity \\
\hline $2^{\text {nd }}$ Human observer & $96.11 \%$ & $67.33 \%$ & $99.38 \%$ \\
\hline $\begin{array}{c}\text { QUARTZ (without } \\
\text { pre/post processing) }\end{array}$ & $95.64 \%$ & $73.66 \%$ & $98.14 \%$ \\
\hline $\begin{array}{c}\text { QUARTZ (with } \\
\text { pre/post processing) }\end{array}$ & $95.84 \%$ & $69.12 \%$ & $98.88 \%$ \\
\hline
\end{tabular}

\section{B. Vessel analysis}

A morphological thinning operation was applied to the segmented vasculature to create centrelines, followed by the removal of spurs, bifurcation and crossover points to create vessel segments. The local orientation of a vessel was calculated by first fitting a parametric spline curve to obtain a smooth centreline. The derivatives of the spline curve were evaluated to compute the vessel orientations (local angle with respect to $\mathrm{x}$-axis) at centreline coordinates [21].

The width of a vessel segment is the distance between the locations of edge points of the vessel segment orthogonal to the vessel centreline orientation, calculated at each centreline coordinate. QUARTZ has included two options for determining the edge points: (i) using zero-crossings of the second derivative following smoothing with an anisotropic Gaussian filter [21], (ii) fitting a 2-D Gaussian function to the local section of the vessel segment and using zerocrossings of the second derivative of the optimized Gaussian curve [34]. The former was used in this study. The centrelines, local orientations, edge points and vessel widths were all calculated with sub-pixel accuracy. Fig.3 illustrates these measures marked onto retinal images.
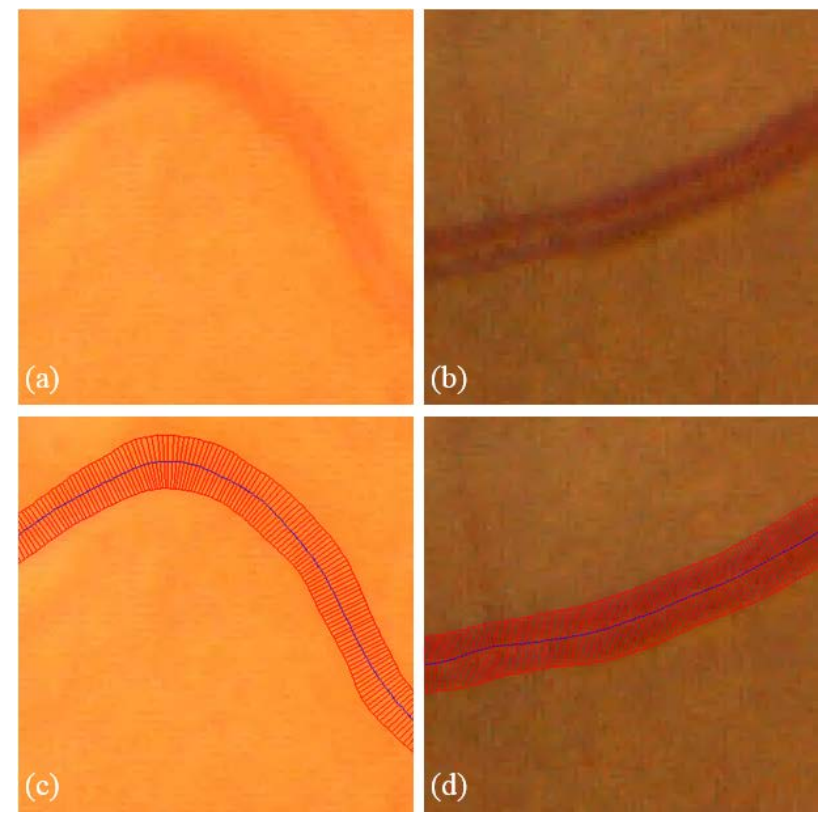

Fig.3. (a)-(b) Zoom-in regions of retinal images. (c)-(d) Vessel centreline, edge, and width markings corresponding to (a)-(b). (c) UK Biobank.

For evaluation, using the 3,351 images of adequate quality, 1992 vessel profiles from different kinds of vessels were selected to compare manual with automated measures. These include: 615 profiles from normal vessel segments, 383 profiles from vessel segments with a central reflex, 344 profiles from vessel segments with uneven illumination and 650 profiles from vessels with normal as well as central reflex along their length. Table 2 shows the mean $(\mu)$ and standard deviation $(\sigma)$ of vessel width measures for the reference standard (mean of the two human observers) and the automated method.

Table 2. The mean and standard deviation of vessel width measures (in pixels).

\begin{tabular}{|l|c|c|c|c|}
\hline \multirow{2}{*}{ Vessel segment type } & \multicolumn{2}{|c|}{ Reference standard } & \multicolumn{2}{c|}{ Automated method } \\
\cline { 2 - 5 } & $\mu$ & $\sigma$ & $\mu$ & $\sigma$ \\
\hline Normal & 10.46 & 1.26 & 10.00 & 1.60 \\
\hline Central reflex & 10.76 & 1.31 & 10.06 & 1.51 \\
\hline Uneven illumination & 11.29 & 0.85 & 10.56 & 1.89 \\
\hline Normal/central reflex & 11.09 & 0.97 & 10.18 & 1.23 \\
\hline For all vessel & 10.87 & 1.16 & 10.17 & 1.54 \\
\hline
\end{tabular}

The overall mean and standard deviation of width measures for all the vessel profiles measured by the automated method is 10.17 and 1.54 pixels respectively, which is similar to that of the reference standard of 10.87 and 1.16 pixels respectively. The differences in vessel width measures comparing the reference standard with the automated method are also important. It should be noted that low variance in width differences might be more important than the absolute difference in measures of width. Any systematic bias in measures of width may be unimportant as long as widths are measured consistently. The mean and standard deviation of differences in the measures computed by the algorithm compared to the reference standard are only 0.70 and 1.13 pixels respectively. These results are comparable with semi-automated approaches, e.g. Trucco [19] reports a standard deviation of differences between 0.319-1.381 assessed on the REVIEW dataset [35].

The full list of quantitative measures computed at this stage for every segment contain: smoothed centreline coordinates of the segment, local orientation angle at each centreline coordinate, width of vessel segment at each centreline coordinate, mean width of the segment, multiple tortuosity measures of the segment and length of the segment. The tortuosity measures include arc/chord ratio, curve energy [36] and level-wise-mean, which is a subdivided chord length method [22].

\section{Arteriole/venule ( $a / v)$ classification}

The vessel segments from the previous section were classified into arterioles and venules using a convolutional neural network whose architecture contains six learned layers: three convolutional and three fully connected [27]. For each centreline pixel in a vessel segment, a small square colour image patch centred on the target pixel was provided as the input for the convolutional neural network in order to assign an arteriole or venule label to the target pixel. The labels for the centreline pixels were then used to award the whole vessel segment as arteriole or venule using a voting strategy.

For a/v classification, 100 images were randomly selected from the 3,351 images of adequate quality. The vessel segments from these images were manually labelled as arteriole or venule by two human observers. The labelled data by the first observer was used as the reference standard. The second observer labelled a random subset of five images; and a high agreement of $98.84 \%$ was achieved between the two observers. All centreline pixels in a segment will take up the manual label awarded to the segment. This equated to 835,914 centreline pixels possessing a manual label. The 100 images were randomly divided into three sets: 50 training images, 15 validation images and 35 testing images. The classifier was built using the image patches derived from the manually labelled centreline pixels of the training set (following data augmentation) and validation set, and 
evaluated using the image patches derived from the manually labelled centreline pixels of the testing set.

The algorithm was evaluated in terms of sensitivity, specificity and accuracy on a per centreline pixel and per segment basis and the results are presented in Table 3. The performance metrics have been stated separately for arterioles and venules. However, this is a single classification problem with two classes, hence sensitivity for arterioles equals specificity for venules and sensitivity for venules equals specificity for arterioles. An accuracy of $86.97 \%$ (per pixel basis) represents a high level of performance when considering the variability in image quality of the dataset. Results are comparable with Trucco [19], with a reported accuracy of 85.47-87.19\% (dataset used not stated). However, their assessment was limited to vessels within a concentric area centred on the optic disc as opposed to the vessels from the entire retinal image. The classification of vessel segments into arterioles and venules is shown in Fig.4, where red colour segments represent arterioles and blue colour segments represent venules.

Table 3. Performance of a/v classification.

\begin{tabular}{|c|c|c|c|}
\hline Level & Measure & Arteriole & Venule \\
\hline \multirow{3}{*}{ Pixel } & Sensitivity & $86.07 \%$ & $87.67 \%$ \\
\cline { 2 - 4 } & Specificity & $87.67 \%$ & $86.07 \%$ \\
\cline { 2 - 4 } & Accuracy & $86.97 \%$ & $86.97 \%$ \\
\hline \multirow{3}{*}{ Segment } & Sensitivity & $85.14 \%$ & $85.32 \%$ \\
\cline { 2 - 4 } & Specificity & $85.32 \%$ & $85.14 \%$ \\
\cline { 2 - 4 } & Accuracy & $85.24 \%$ & $85.24 \%$ \\
\hline
\end{tabular}
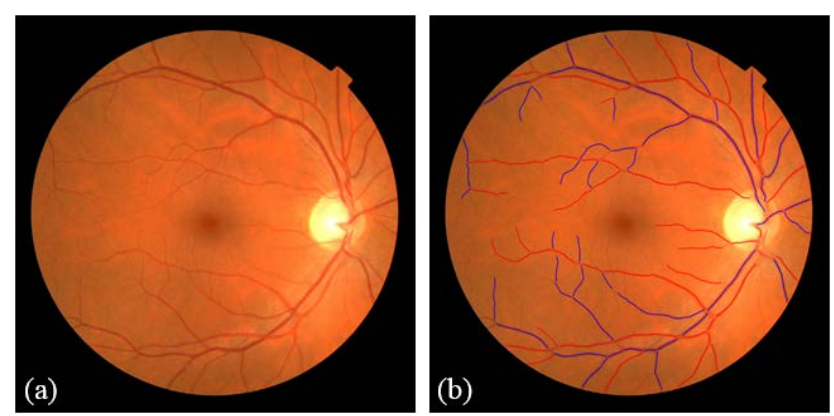

Fig. 4. Classification of arterioles and venules. (a) Original retinal image. (b) Classification results. (C) UK Biobank

\section{Optic disc localization}

A simple and robust optic disc localization algorithm was implemented into QUARTZ. Shade correction was performed by subtracting an image approximating the background (approximated using a median filter with a large kernel size). The location of maximum intensity was identified and was regarded as a point within the optic disc. A condition was set that restricted the search region to large circular regions on the left and right side of the image.

For evaluation, 500 images were randomly selected from the 3,351 images of adequate quality. 488 out of 500 optic disc locations were correctly detected (pixel location within the optic disc boundary decided by human observers), equating to a success rate of $97.60 \%$. This is an impressive detection rate considering the variability in quality of images in the dataset. Fig.5 illustrates examples of successful optic disc detection. This result compares favourably with literature, with Niemeijer [37] reporting success rates between 93.0\% and $99.4 \%$ for a challenging dataset.
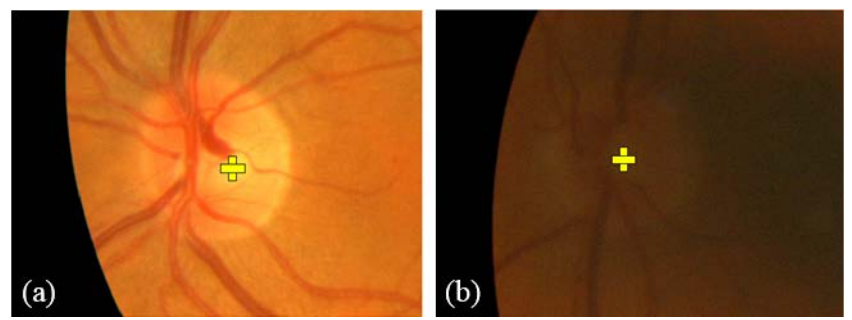

Fig. 5. Optic disc localization from retinal images of variable illumination conditions. (C) UK Biobank.

\section{E. Image quality assessment}

Large retinal datasets used in epidemiological studies contain relatively high proportion of images of poor quality. However, useful information can be extracted from well segmented sections of the vasculature, even if this only represents a portion of the vascular tree. For example, images with uneven illumination are still likely to present at least a section of the retina with sufficient illumination to allow for accurate segmentation. This approach ensures that there is little wastage by making use of many of the poor images, extracting as much information as possible from these retinal datasets.

Manual assessment of the segmentation would see images rejected if less than half the vasculature was segmented, if the segmentation was considered fragmented/unconnected and if multiple non-vessel objects were segmented. An automated image quality assessment algorithm [26] was designed to mimic this. Global features of area, fragmentation and complexity measured from the segmented vessel map and support vector classification were used to label the images as inadequate or adequate.
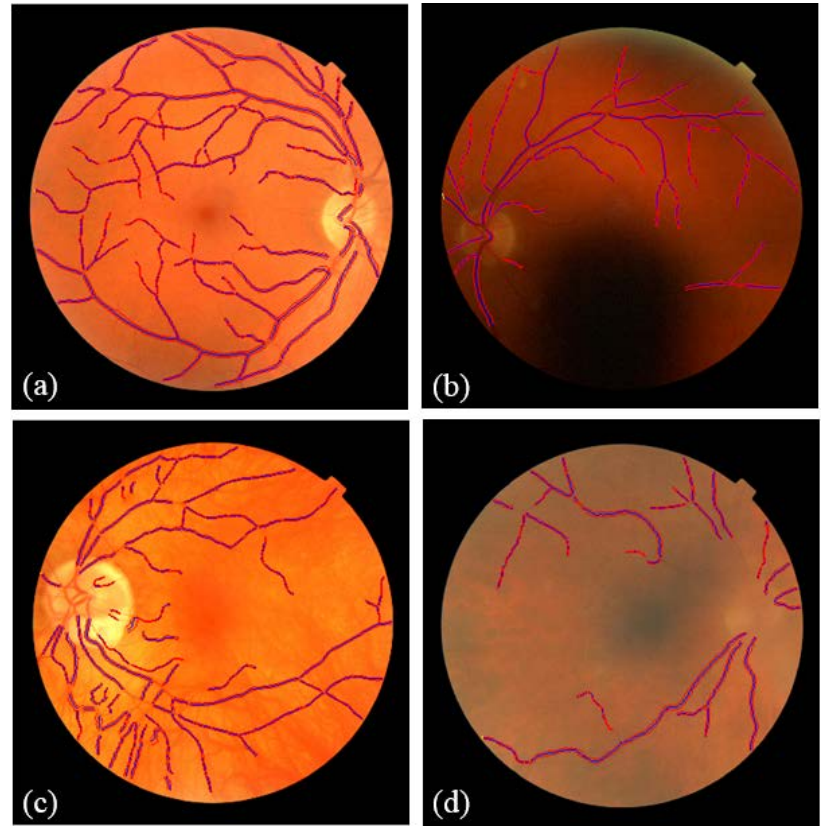

Fig. 6. Images correctly classified as (a)-(b) adequate quality, (c)(d) inadequate quality. (c) UK Biobank.

For evaluation, 1,000 images were randomly selected from the complete subset of 4,692 images. Manual image quality assessment was performed on the segmentation of these images, 240 were manually labelled as inadequate and 760 were labelled as adequate. Two human observers were used for manual labelling. The labelling stated above was performed by the first observer and was used as the reference standard. The second observer labelled a random subset of 100 images out of the 1,000. A high agreement of $99 \%$ was achieved 
between the two observers, which aided the validation of the reliability of the manual labels.

The 1,000 manually labelled images were randomly divided into two sets, each containing 500 images. The classifier was trained on one set and tested on the other set. The performance of the algorithm for the detection of images of inadequate quality achieved a sensitivity of $95.00 \%$ and a specificity of $93.95 \%$. This equates to $72.60 \%$ of the 500 images being labelled as adequate, of which $98.35 \%$ were correctly identified of adequate quality. When applied to the whole subset of 4,692 images, this resulted in 3,351 images (71.42\%) being labelled as adequate. Fig. 6 shows examples of images successfully classified as adequate and inadequate. Fig. 6(c) contains the false segmentation of choroidal vessels and Fig. 6(d) has insufficient illumination/contrast.

QUARTZ also exports an image quality score for each image, which is a value ranging from 0 to 1 (derived using the distance to the decision boundary of the classifier). Note, the performance stated above equates to images being labelled as inadequate if the quality score $<=0.6033$.

\section{PROCESSING 136,000 IMAGES}

Following the successful validation of the modules of QUARTZ using a subset of 4,692 images, the entire retinal dataset $(135,867$ retinal images) from the UK Biobank at baseline examination was automatically processed. This resulted in 97,188 (71.53\%) images classified as being of adequate quality, which included 55,457 (80.90\%) participants with at least one image included. A full breakdown of participant numbers is provided in Table 4 .

Table 4. Proportions of participants with images classified as adequate quality for inclusion in vessel morphometric data suitable for epidemiological studies.

\begin{tabular}{|l|l|}
\hline Participants with no images included & $\begin{array}{l}13,092 / 68,549 \\
(19.10 \%)\end{array}$ \\
\hline Participants with one image included & $13,726 / 68,549$ \\
& $(20.02 \%)$ \\
\hline Participants with two images included & $41,731 / 68,549$ \\
& $(60.88 \%)$ \\
\hline Participants with at least one image included & $\begin{array}{l}55,457 / 68,549 \\
(80.90 \%)\end{array}$ \\
\hline Number of retinal images included & $97,188 / 135,867$ \\
& $(71.53 \%)$ \\
\hline
\end{tabular}

On a standard machine (i7 processor, 8GB RAM, Nvidia Quadro K1100M GPU) one image can be processed in 2 minutes. Therefore, this would take 6 months to process the entire dataset on a single machine. This can be reduced significantly by the use of a computing cluster.

\section{DISCUSSION AND CONCLUSION}

In this paper, we have presented validation of the retinal image analysis system, QUARTZ, which is designed for the automated processing of large numbers of retinal images and obtains quantitative measures of vessel morphology to be used in epidemiological studies. The system's modules have been validated using a subset of 4,692 images from the UK Biobank retinal image dataset, showing a high level of performance. The robustness of the modules and the fully automated framework made it practical to run on the entire UK Biobank retinal dataset at baseline examination $(135,867$ retinal images). To the best of our knowledge, our research group are the first to report on the completion of this task and the first to report on processing of retinal images on this scale. Also, the output is maximised by extracting information from the entire retinal image and not just concentric rings. The UK Biobank presents a considerably amount of variability in respect to the quality of images. This was tackled with a robust vessel segmentation procedure combined with an effective image quality assessment approach (minimise wastage). The resultant was a high percentage of images (71.53\%) being deemed as adequate for use in the creation of vessel morphometric data. Approximately $98.35 \%$ were correctly identified to be of adequate quality.

Approximately half of the images in the UK Biobank retinal image dataset can be considered as high quality. With $28.47 \%$ of images being automatically rejected, there still remains a high level of variability within the accepted images due to efforts to minimize wastage by effectively making use of many of the poor images. Therefore, it is essential that all of the system's modules are robust and the multiple evaluations presented in this paper successfully demonstrate this. QUARTZ achieves a vessel segmentation accuracy of $95.84 \%$, an optic disc detection success rate of $97.60 \%$, an a/v classification accuracy of $86.97 \%$ and a standard deviation of vessel width differences of 1.13 pixels. These figures refer to evaluation using images within the subset that were automatically deemed to be of adequate quality.

QUARTZ will be used to process retinal images from follow up health checks (once made available by UK Biobank); measuring changes in vessel morphology may also be an important biomarker. Also, participants who did not have retinal images captured at the baseline examination may have retinal images captured at the repeat examination. Therefore, extending processing to the repeat examination dataset has the potential to increase the total sample size.

In conclusion, $71.53 \%$ of the UK Biobank retinal database amounts to a large number of retinal images $(97,188$ images from 55,457 participants) being suitable for analysis. This equates to $80.90 \%$ of participants with at least one adequate quality image (in the assessment of CVD risk, information is only required from one image per person). This, along with the fact that the quantitative measures of vessel morphology can be reliably provided by QUARTZ and then studied in association with a range of medical information obtained from multiple health checks of the participants, makes this a valuable source of data. The vessel morphometric data obtained from the application of QUARTZ on the UK Biobank retinal database are currently being used in epidemiological studies, relating vessel morphology to disease risk factors and outcomes, with publications to follow in the near future.

The intention of the UK Biobank Eye and Vision Consortium is to include this vessel morphometric data in the UK Biobank data archive.

\section{MEMBERS OF THE UK BIOBANK EYE AND VISION CONSORTIUM}

Prof Tariq Aslam, Prof Sarah Barman, Prof Paul Bishop , $\mathrm{Mr}$ Peter Blows, Dr Catey Bunce, Dr Roxana Carare, Prof Usha Chakravarthy, Miss Michelle Chan, Mrs Antonietta Chianca, Dr Valentina Cipriani, Prof David Crabb, Mrs Philippa Cumberland, Dr Alexander Day, Miss Parul Desai, Prof Bal Dhillon, Prof Andrew Dick, Prof Paul Foster, Dr John Gallacher, Prof David (Ted) GarwayHeath, Mr Srini Goverdhan, Prof Jeremy Guggenheim, Mrs Priyal Gupta, Prof Chris Hammond, Dr Ruth Hogg, Prof Anne Hughes, Mr Pearse Keane, Prof Sir Peng Tee Khaw, Mr Anthony Khawaja, Mr Gerassimos Lascaratos, Prof Andrew Lotery, Prof Phil Luthert, Dr Tom Mac-Gillivray, Dr Sarah Mackie, Prof Keith Martin, Ms Michelle McGaughey, Dr Bernadette McGuinness, Dr Gareth McKay, 
Mr Martin McKibbin, Dr Danny Mitry, Prof Tony Moore, Prof James Morgan, Ms Zaynah Muthy, Mr Eoin O'Sullivan, Prof Chris Owen, Mr Praveen Patel, Dr Tunde Peto, Prof Jugnoo Rahi, Dr Alicja Rudnicka, Miss Carlota Grossi Sampedro, Mr David Steel, Mrs Irene Stratton, Mr Nicholas Strouthidis, Prof Cathie Sudlow, Dr Caroline Thaung, Miss Dhanes Thomas, Prof Emanuele Trucco, Mr Adnan Tufail, Prof Stephen Vernon, Mr Ananth Viswanathan, Miss Cathy Williams, Dr Katie Williams, Prof John Yates, Dr Max Yates, Dr Jennifer Yip, Dr Haogang Zhu.

\section{ACKNOWLEDGMENT}

This research has been conducted using the UK Biobank resource under application number 522. The UK Biobank Eye and Vision Consortium is supported by funding from The Special Trustees of Moorfields Eye Hospital NHS Foundation Trust, and at the NIHR Biomedical Research Centre at Moorfields Eye Hospital and UCL Institute of Ophthalmology. The image analysis work was supported by the Medical Research Council Population and Systems Medicine Board (MR/L02005X/1), the British Heart Foundation (PG/15/101/31889), and Fight for Sight (1477/8).

\section{REFERENCES}

[1] Office for National Statistics, Deaths registered in England and Wales in 2013, by cause, (2014).

[2] K. Smolina, F.L. Wright, et al., Incidence and 30-day case fatality for acute myocardial infarction in England in 2010: national-linked database study, Eur. J. Public Health, 22 (2012) 848-853.

[3] K. Smolina, F.L. Wright, et al., Determinants of the decline in mortality from acute myocardial infarction in England between 2002 and 2010: linked national database study, BMJ, 344 (2012) d8059.

[4] S.R. Seshasai, S. Kaptoge, et al., Diabetes mellitus, fasting glucose, and risk of cause-specific death, N. Engl J. Med. 364 (2011) 829-841.

[5] G.S. Collins and D.G. Altman, An independent and external validation of QRISK2 cardiovascular disease risk score: a prospective open cohort study, BMJ, 340 (2010) c2442.

[6] J. Hippisley-Cox, C. Coupland, et al., Derivation, validation, and evaluation of a new QRISK model to estimate lifetime risk of cardiovascular disease: cohort study using QResearch database, BMJ, 341 (2010) c6624. [7] S.G. Wannamethee, P. Welsh, et al., N-terminal pro-brain natriuretic Peptide is a more useful predictor of cardiovascular disease risk than Creactive protein in older men with and without pre-existing cardiovascular disease, J. Am. Coll. Cardiol. 58 (2011) 56-64.

[8] N. Cheung, S.M. Saw, et al., BMI and retinal vascular caliber in children, Obesity, 15 (2007) 209-215.

[9] C.G. Owen, A.R. Rudnicka, et al., Retinal arteriolar tortuosity and cardiovascular risk factors in a multi-ethnic population study of 10-year-old children; the Child Heart and Health Study in England (CHASE), Arterioscler. Thromb. Vasc. Biol. 31 (2011) 1933-1938.

[10] T.Y. Wong, F.M. Islam, et al., Retinal vascular caliber, cardiovascular risk factors, and inflammation: the multi-ethnic study of atherosclerosis (MESA), Invest. Ophthalmol. Vis. Sci. 47 (2006) 2341-2350.

[11] T.Y. Wong, R. Klein, et al., Retinal arteriolar narrowing and risk of coronary heart disease in men and women: the Atherosclerosis Risk in Communities Study, JAMA, 287 (2002) 1153-1159.

[12] M.D. Abràmoff, M.K. Garvin, et al., Retinal imaging and image analysis, IEEE Rev. Biomed. Eng. 3 (2010) 169-208.

[13] C.Y. Cheung, M.K. Ikram, et al., The clinical implications of recent studies on the structure and function of the retinal microvasculature in diabetes, Diabetologia, 58 (2015) 871-885.

[14] M.K. Ikram, Y.T. Ong, et al., Retinal vascular caliber measurements: clinical significance, current knowledge and future perspectives, Ophthalmologica, 229 (2013) 125-136.

[15] UK Biobank, http://www.ukbiobankeyeconsortium.org.uk/.

[16] M.M. Fraz, R.A. Welikala, et al., QUARTZ: Quantitative Analysis of Retinal Vessel Topology and size - An automated system for quantification of retinal vessels morphology, Expert Syst. Appl. 42 (2015) 7221-7234.
[17] T.J. MacGillivray, J.R. Cameron, et al., Suitability of UK Biobank retinal images for automatic analysis of morphometric properties of the vasculature, PLoS ONE 10 (2015) e0127914.

[18] A. Perez-Rovira, T. MacGillivray, et al., VAMPIRE: Vessel Assessment and Measurement Platform for Images of the REtina, Engineering in Medicine and Biology Society, EMBC, International Conference of the IEEE (2011) 3391-3394.

[19] E. Trucco, L. Ballerini, et al., Novel VAMPIRE algorithms for quantitative analysis of the retinal vasculature, Biosignals and Biorobotics, (2013) 1-4.

[20] M. Ortega, N. Barreira, et al., Sirius: A web-based system for retinal image analysis, Int. J. Med. Inform. 79 (2010) 722-732.

[21] P. Bankhead, C.N. Scholfield, et al., Fast retinal vessel detection and measurement using wavelets and edge location refinement, PLOS ONE 7 (2012) e32435.

[22] C.G. Owen, A.R. Rudnicka, et al., Measuring retinal vessel tortuosity in 10-year-old children: validation of the computer-assisted image analysis of the retina (CAIAR) program, Invest. Ophthalmol. Vis. Sci. 50 (2009) 2004-2010. [23] C.M. Wilson, K.D. Cocker, et al., Computerized analysis of retinal vessel width and tortuosity in premature infants, Invest. Ophthalmol. Vis. Sci. 49 (2008) 3577-3585.

[24] S.G. Vázquez, B. Cancela, et al., Improving retinal artery and vein classification by means of a minimal path approach, Mach. Vis. Appl. 24 (2013) 919-930.

[25] M. Yates, S. Hayat, et al., Retinal Vessel Morphometry Associations with Polymyalgia Rheumatica; Findings from the European Prospective Investigation of Cancer (EPIC) in Norfolk [abstract], Arthritis Rheumatol. 10 (2016).

[26] R.A. Welikala, M.M. Fraz, et al., Automated retinal image quality assessment on the UK Biobank dataset for epidemiological studies, Comput. Biol. Med. 71 (2016) 67-76.

[27] R.A. Welikala, A.R. Rudincka, et al., Automated arteriole and venule classification in retinal images using deep learning, Comput. Biol. Med. (submitted).

[28] U.T.V. Nguyen, A. Bhuiyan, et al., An effective retinal blood vessel segmentation method using multi-scale line detection, Pattern Recognit. 46 (2013) 703-715

[29] M.M. Fraz, P. Remagnino, et al., An ensemble classification-based approach applied to retinal blood vessel segmentation, IEEE Trans. Biomed. Eng. 59 (2012) 2538-2548.

[30] D. Marín, A. Aquino, et al., A new supervised method for blood vessel segmentation in retinal images by using gray-level and moment invariantsbased features, IEEE Trans. Med. Imaging, 30 (2011) 146-158.

[31] J.V.B. Soares, J.J.G. Leandro, et al., Retinal vessel segmentation using the 2-D Gabor wavelet and supervised classification, IEEE Trans. Med. Imaging, 25 (2006) 1214-1222.

[32] J. Staal, M.D. Abràmoff, et al., Ridge-based vessel segmentation in color images of the retina, IEEE Trans. Med. Imaging, 23 (2004) 501-509.

[33] A.D. Hoover, V. Kouznetsova, et al., Locating blood vessels in retinal images by piecewise threshold probing of a matched filter response, IEEE Trans. Med. Imaging, 19 (2000) 203-210.

[34] M.M. Fraz, P. Remagnino, et al., Quantification of blood vessel calibre in retinal images of multi-ethnic school children using a model based approach, Comput. Med. Imaging Graph. 37 (2013) 48-60.

[35] B. Al-Diri, A. Hunter, et al., REVIEW-a reference data set for retinal vessel profiles, Engineering in Medicine and Biology Society, EMBC, International Conference of the IEEE, (2008) 2262-2265.

[36] C.G. Owen, R.S.B. Newsom, et al., Diabetes and the tortuosity of vessels of the bulbar conjunctiva, Ophthalmology, 115 (2008) 27-32.

[37] M. Niemeijer, M.D. Abràmoff, et al., Fast detection of the optic disc and fovea in color fundus photographs, Med. Image Anal. 13 (2009) 859-870. 